\title{
Segmental volume changes that occur in nonhuman primates during short term head up (HUT) and head down (HDT) tilt
}

\author{
Leslie David Montgomery ${ }^{1,3}$ and Clarence Oloff ${ }^{2}$ \\ 1. LDM Associates, San Jose, USA \\ 2. Retired \\ 3. E-mail any correspondence to: pmontgomery@telis.org
}

\begin{abstract}
Nonhuman primates are often used in biomedical research and to investigate physiologic processes that occur in man. Impedance plethysmography was used to measure calf, thigh, pelvic, abdominal, and thoracic volume changes in ten Rhesus and eight squirrel monkeys during five-minute exposures to HUT and HDT at angles of 5, 10, and 20 degrees. Calf, rump and tail measurements were made in three squirrel monkeys at 10 and 20 degrees of HUT and HDT. Fluid volume changes in all segments of the Rhesus monkeys were found to change during HUT an HDT in direct relation to the angle of tilt used. However, the volume changes that occurred in the squirrel monkeys were found to be quite different. Their calf, thigh, and pelvic segments lost volume during both HUT and HDT while their abdominal and thoracic segments responded similarly to those of the Rhesus monkeys. These results and those of the calf/tail measurements of the squirrel monkeys suggest that they may utilize their tails as a compensatory reservoir during postural changes and therefore, may not be an appropriate animal model for man under some orthostatic test conditions.
\end{abstract}

Keywords: Bioimpedance; Monkeys; Head-up tilt; Head-down tilt

\section{Introduction}

Nonhuman primates are often used in biomedical research, clinical investigations, and drug development and behavioral studies [1,2]. For the most part, the nonhuman primates of choice are the Rhesus (Macaca Mulatta) [3] and squirrel (Saimiri sciurea) [4] monkeys. Rhesus monkeys are used because of their similarity to man and due to the extensive amount of background information available on this species. Squirrel monkeys are smaller, less expensive, and easier to handle and maintain in smaller facilities.

Both the Rhesus and squirrel monkeys have been used in blood pressure research [5-7], locomotion and postural behavior studies [8], stability during ambulation [9] and aerospace physiology [10]. Fluid redistribution between different body segments during postural changes may be an influencing factor in all of these cases. However, the fluid redistribution in the Rhesus monkey may be quite different from that found in the squirrel monkey under similar conditions.

One source of possible difference between the fluid responses of the two species during postural changes may be the differences exhibited during locomotion in their natural environments. Rhesus monkeys prefer open grassland and woodland habitats. They spend a large part of their time sitting on the ground in a semi-upright posture and walk or run in a terrestrial quadrupdal manner. Squirrel monkeys, by comparison, prefer the primary and secondary forest, riverine forest and understory as their natural habitat. They are only occasionally on the ground. Their preferred form of locomotion is active arboreal quadrupedalism when walking, running and leaping. [7].

To the best of our knowledge, no studies have made direct comparisons of the Rhesus and squirrel monkeys regarding possible segmental fluid redistribution as a component in locomotion, blood pressure control or balance when they change positions, undergo orthostatic stress or travel along tree branches. To quote Alan G. Brady [5] directly: 


\begin{abstract}
"To properly utilize these animals (squirrel monkeys) it is important that research techniques are properly suited to the unique characteristics of this genus. To consider squirrel monkeys as 'miniaturized macaques', or a slightly different version of another animal model, is to underestimate both the potential and pitfalls of using these animals in research."
\end{abstract}

With this quote in mind, we measured the fluid volume responses in the upper and lower body segments of both the Rhesus and squirrel monkeys during short-term head up and head down tilt. It is our hope that this information will be useful in biomedical research and behavioral studies.

\section{Materials and Methods}

Ten male Rhesus and eight male squirrel monkeys were exposed to head up and head down tilt table orthostatic test sequences of 5, 10, and 20 degrees. Each animal was anesthetized, instrumented, and allowed to stabilize for thirty minutes prior to each tilt table test sequence. Each test sequence consisted of a five-minute control period and five minutes exposure at each tilt angle separated by a fiveminute horizontal period and followed by a five-minute horizontal recovery period. Impedance plethysmography was used to monitor segmental volume changes every 15 sec. during each test sequence. The calf, thigh, pelvic, abdominal, and thoracic volume responses were measured during the squirrel monkey test. Calf, thigh, pelvic, abdominal, thoracic, and arm volume changes were measured during the Rhesus monkey tests.

Each head up tilt (HUT) test sequence consisted of the following position/time sequence:

\begin{tabular}{lll}
\hline Position & $\begin{array}{l}\text { Elapsed time } \\
(\min )\end{array}$ & $\begin{array}{l}\text { Time at position } \\
(\min )\end{array}$ \\
\hline Flat & 5 & 5 \\
+5 & 10 & 5 \\
Flat & 15 & 5 \\
+10 & 20 & 5 \\
Flat & 25 & 5 \\
+20 & 30 & 5 \\
Flat & 35 & 5 \\
\hline
\end{tabular}

Each head down tilt (HDT) test sequence was similar to the one above with the animals at $-5,-10$, and -20 degrees.

Details of the experimental protocol are given below.

\section{Description of impedance plethysmography}

The impedance plethysmograph measures the electrical impedance changes that vary with the blood/fluid content of a body segment during each blood pulse. With a tetrapolar arrangement, four electrodes are placed on or around the given segment, separated by various axial distances. A high-frequency, low-amperage electrical current is applied between the outer two electrodes. Simultaneously, the resistance produced by the electrically conductive tissue and blood is measured between the two inner electrodes. Records of segmental base resistance can be analyzed according to Nyboer [11] to determine the amount of blood accumulated in the body segment during various stress sequences. Values of segmental blood pooling may be expressed in terms of relative $(\mathrm{ml} / 100 \mathrm{ml}$ of body tissue) or absolute (ml) volume accumulation. Additional blood accumulating between the two inner electrodes during a pulse has the effect of placing a second resistor in parallel with the tissue resistance lying between the two recording electrodes. This, in turn, decreases the total segmental resistance between the recording electrodes proportionately to the pulse blood flow. These pulsatile segmental resistance changes can be analyzed to obtain the total $(\mathrm{ml} / \mathrm{min})$ or relative $(\mathrm{ml} / 100 \mathrm{ml}$ of body tissue/min) blood flow through the segment.

\section{Experimental method}

Conditions

All observations were made at an average ambient temperature of $21.0 \pm 0.5 \mathrm{C}$ with the animals resting in either a head-up or antiorthostatic (head-down tilt) position. Air movement within the room was negligible. Bright light, excessive noise or other vasoactive stimulation was kept to a minimum during each test sequence.

\section{Segmental Volume}

Measurements of baseline resistances $\left(R_{0}\right)$ and resistance changes $(\Delta R)$ of the calf, thigh, pelvic, abdominal, thoracic body segments were made during each test sequence using an impedance plethysmograph (UFI Inc. Model 2994, Morro Bay, CA).

Disposable EKG electrodes were attached on the foot, ankle, knee, and midthigh (opposite the groin) region of each animal's leg; on the same side of the body at the iliac crest; on the back of the hand; and on the shoulder during the Rhesus monkey tests. Attempts to use disposable EKG surface electrodes on the tails of the squirrel monkeys proved to be difficult (See "Limitations" section below). Therefore, 26 gauge hypodermic needles were used as percutaneous electrodes at the described sites. Prior to instrumentation, each animal was anesthetized with Ketamine by the attendant veterinarian.

The impedance plethysmograph introduced a highfrequency $(50 \mathrm{kHz})$, low amperage $(0.1 \mathrm{~mA} \mathrm{rms})$ constant current signal between the foot and hand electrodes. $R_{0}$ values were then measured in each segment every two minutes throughout each test sequence. Records of $R_{0}$ at each time were then used to determine the $\Delta R$ responses of each segment during each test sequence relative to that at elapsed time zero.

The measured resistance of the given body segment at any point in time is directly related to segmental length and 
a resistivity factor and inversely related to the average segmental cross-sectional area; which may be written:

$$
\mathrm{R}=\frac{\rho \cdot \mathrm{L}}{\mathrm{A}}
$$

where:

$\rho=$ electrical resistivity of the material in $(\Omega \cdot \mathrm{cm})$

$L=$ length of body segment in $(\mathrm{cm})$

$A=$ average cross-sectional area of segment in $\left(\mathrm{cm}^{2}\right)$

Multiplying this expression by unity in the form $L / L$ and equating the denominator to volume, since $V=A \cdot L$, Eq. (1) may be written as:

$$
\mathrm{R}=\frac{\rho \cdot L^{2}}{\mathrm{~V}}
$$

As explained by Nyboer [11], if the length of the body segment is considered to remain constant during a short term experimental treatment and any change in the segmental volume is considered to be caused by a change in the average cross- sectional area, then any change in segmental volume, $(\Delta V)$ is inversally proportional to a measured change in segmental resistance $(\Delta R)$.

Mathematically:

$$
\Delta \mathrm{R}=R_{1}-R_{2}=\rho \cdot L^{2}\left(1 / V_{1}-1 / V_{2}\right)
$$

or

$$
\begin{gathered}
\Delta \mathrm{R}=\rho \cdot L^{2}\left(1 / V_{1}-1 / V_{2}\right) \\
\Delta \mathrm{R}=-\rho \cdot L^{2}\left(\Delta V / V_{1} \cdot V_{2}\right)
\end{gathered}
$$

which by substitution,

$$
\Delta \mathrm{R} \sim-\rho \cdot L^{2}\left(\Delta V / V^{2}\right) \sim-R(\Delta V / V)
$$

Thus, Equation 3 provides the relation between small changes in volume and the corresponding changes in electrical resistance of the given body segment. In this way, a fractional change in segmental volume is closely related to the fractional change in measured resistance, which may be expressed by the ratio

$$
\frac{V}{V_{0}}=-\frac{\mathrm{R}}{R_{0}}
$$

Using Equation 4, records of segmental base resistance can be analyzed to obtain an index of the percentage change in segmental volume that takes place during various stress sequences.

Base resistance values were recorded on a laptop computer for later analysis. Each body segment was sampled in sequential order by manually positioning a thumb wheel switch to the desired segment number. To identify the given segment on the recorded records, a simultaneous voltage level was manually selected and recorded in parallel as the segment number was changed. This process was repeated every five seconds for the total test period.

During these tests, the animals were placed on their right sides with their tails parallel to their legs. Their tails were elevated and straightened to be parallel to the tabletop during each test position.

\section{Anesthetic}

Each animal was given an initial intramuscular injection of $20 \mathrm{mg} / \mathrm{kg}$ ketamine prior to each tilt table test sequence. Subsequent anesthetic was given through intravenous infusion of $100 \mathrm{mg} / 10 \mathrm{ml}$ ketamine/saline (approximately $0.67 \mathrm{mg} / \mathrm{kg} / \mathrm{min}$ ) over the one-hour period needed to complete the measurement of the desired parameters. Exact dosage and adjustments were determined for each animal by appropriate Veterinary Sciences personnel.

\section{Ethical approval}

The research related to animals use has been complied with all the relevant national regulations and institutional policies for the care and use of animals.

\section{Results}

The results of this study are presented below in Figures 1, 2, and 3 . The $X$-axis of each panel of these figures is the elapsed time ( $\mathrm{min})$ and the $\mathrm{Y}$-axis of each panel is the relative resistance of each segment at time $=0$ (Ohms) divided by the resistance at each subsequent time step. Please note that an increase in segmental (normalized) resistance indicates a corresponding decrease in segmental volume. Conversely, a decrease in segmental resistance indicates an increase in segmental volume.

Figure 1 illustrates the thoracic (THO) and abdominal $(A B)$ upper body volume responses of the squirrel (SQ-left panels) and Rhesus (RH-right panels) monkeys during the head up (U) and head down (D) test sequences.

Figure 2 uses the same format to present the pelvic (PEL), thigh (THI) and calf (CA) lower body volume responses of the squirrel and Rhesus monkeys.

Figure 3 shows the calf (CA), rump (RUMP), and tail (TAIL) volume changes that took place in three squirrel monkeys $(A, B, C)$ during head up $(U)$ and head down (D) tilt at angles of 10 and 20 degrees.

\section{Discussion}

Figures 1 and 2 show the differential fluid redistribution effects of the head up vs. the head down stress periods and indicate differences that may exist regarding how each type of monkey responds to position changes. 
Figure 1: Squirrel (SQ) and Rhesus (RH) monkey thoracic (THO) and abdominal ( $A B$ ) upper body volume changes during short-term head up $(U)$ and head down $(D)$ tilt. See text for explanation.
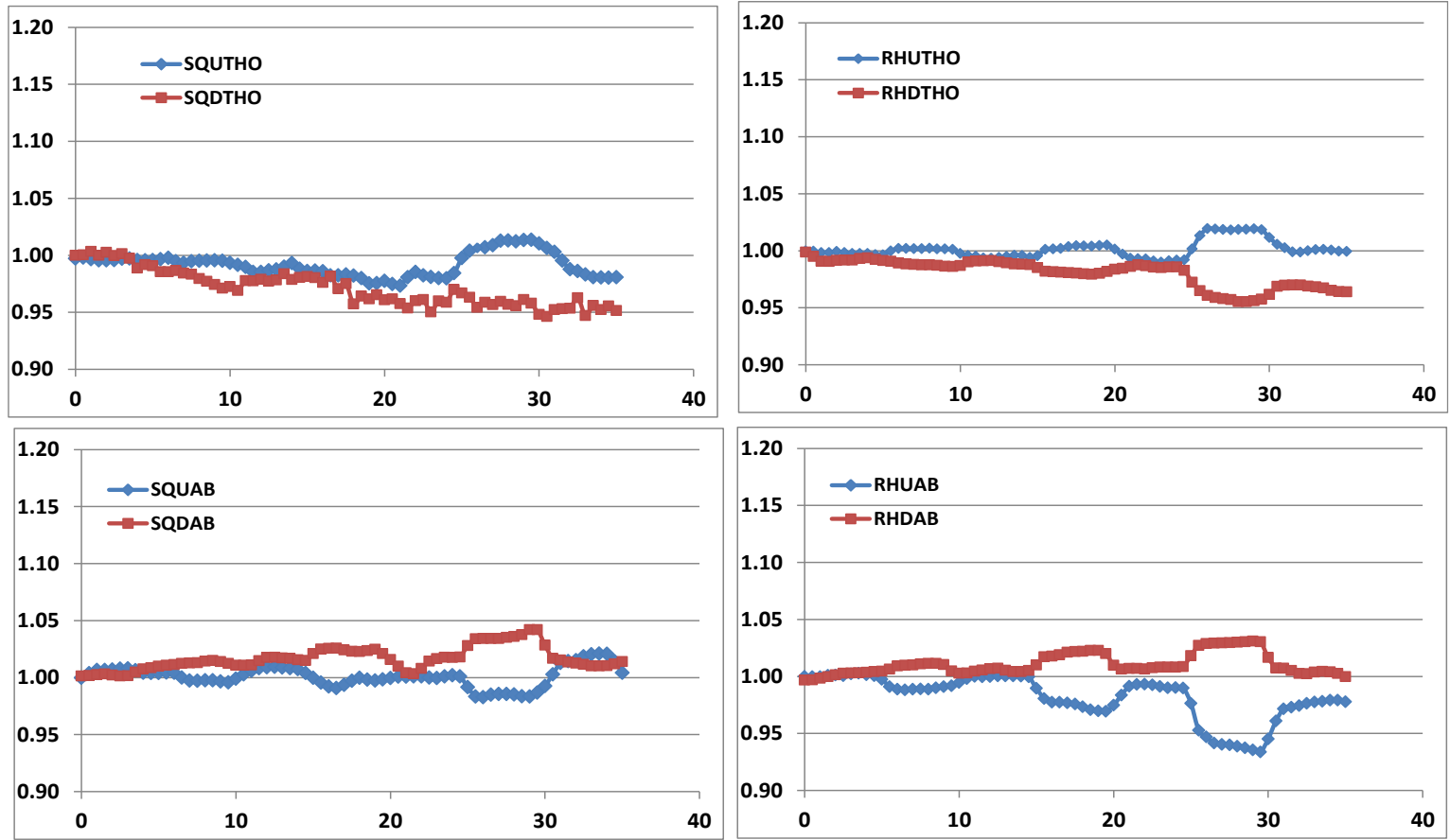

Figure 2: Squirrel and Rhesus monkey pelvic (PEL), thigh (THI), and calf (CA) lower body volume changes during short term head up and head down tilt. See text for explanation.
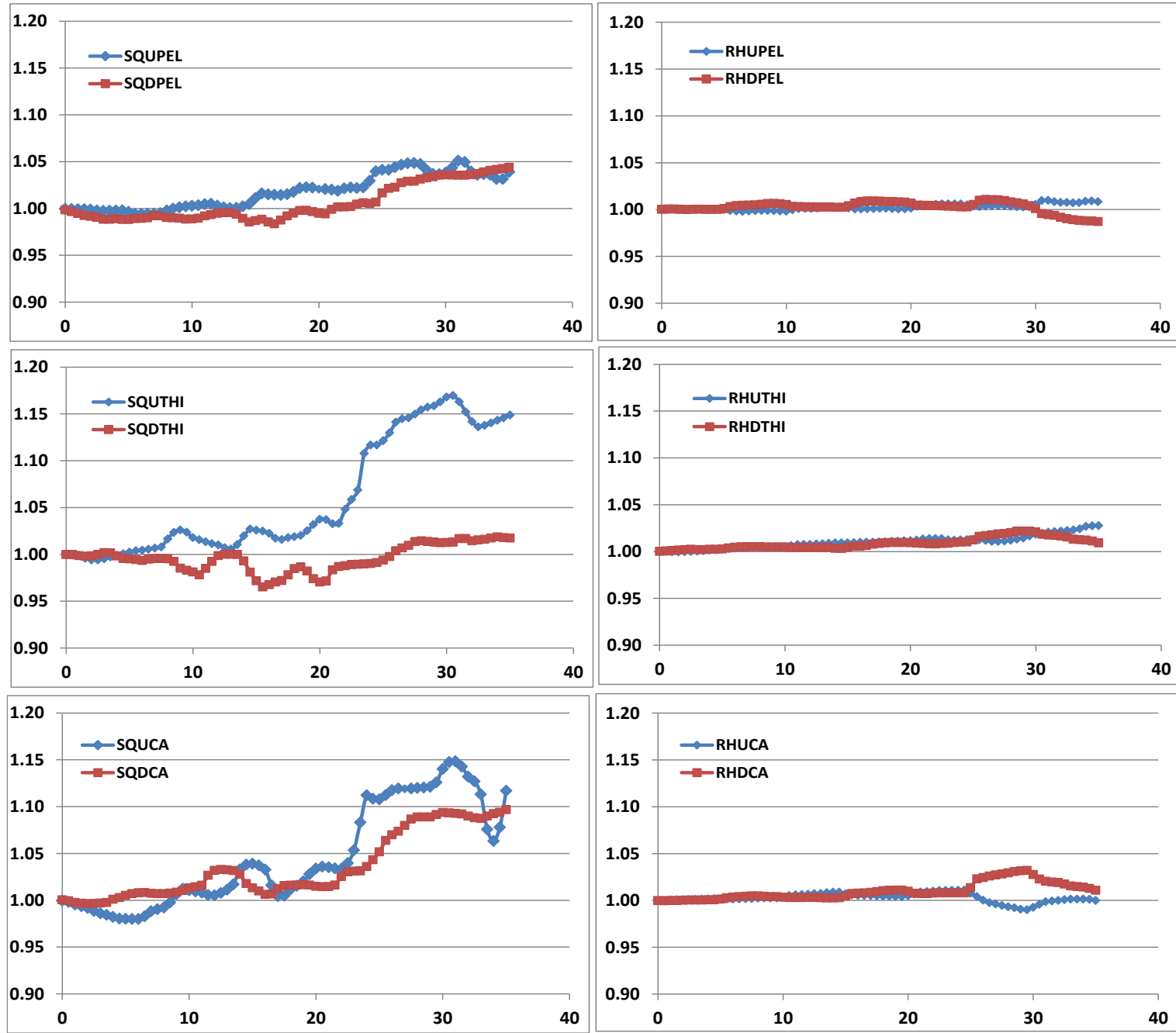
Figure 3: Squirrel monkey calf (CA), rump (RUMP), and tail (TAIL) volume changes during short term head up $(U)$ and head down (D) tilt for monkeys $A, B$, and C. See text for explanation
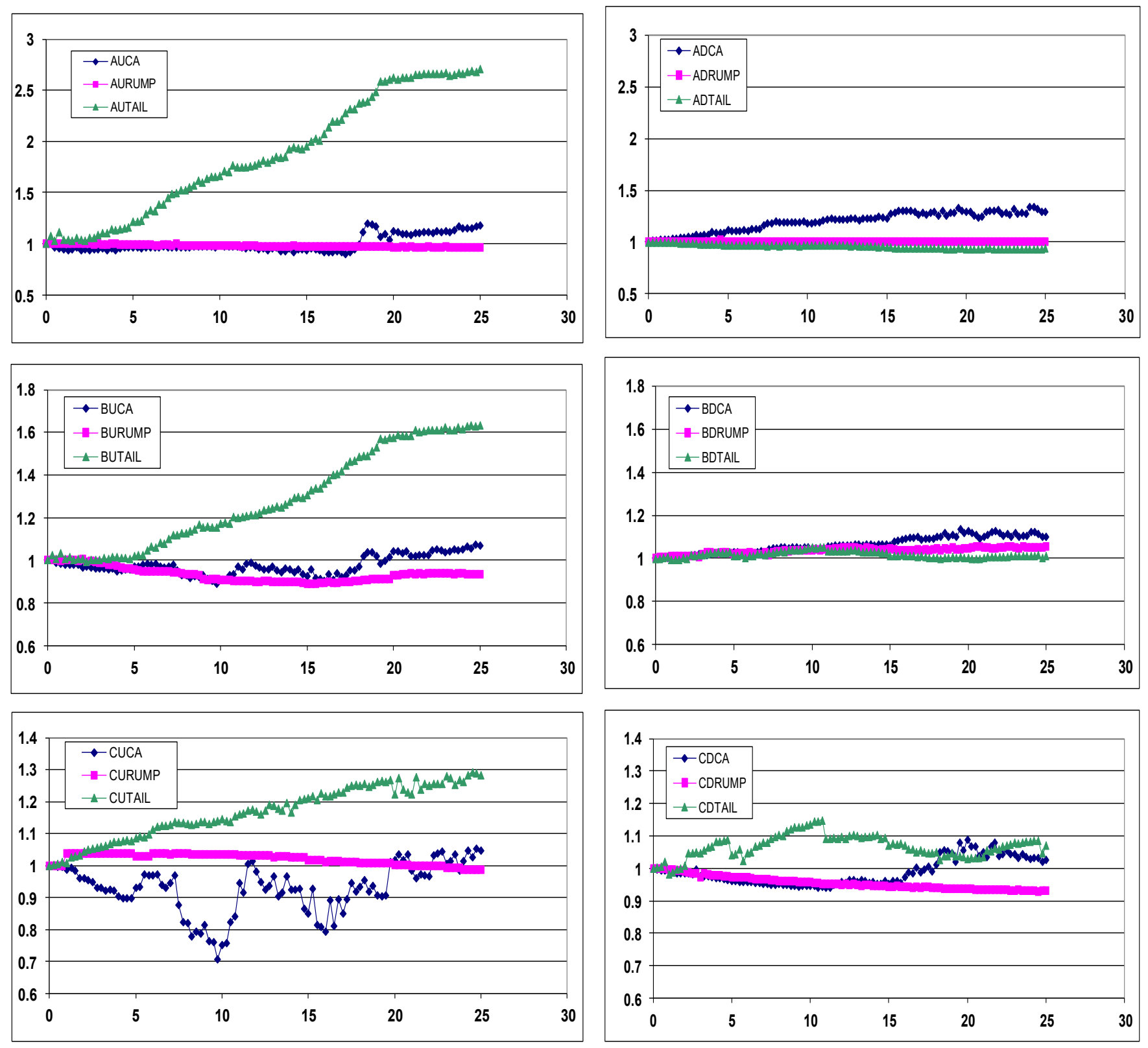

Figures 1 and 2 also show that the amount of fluid transferred between the various body segments is generally graded as a function of the angle of exposure.

Figures 1 and 2 give the percent mean segmental resistance values, relative to the value at elapsed time 0 , for each Rhesus and squirrel monkey body segment measured during both the head up (diamond points) and head down (square points) tests. Segmental resistance, as measured by the impedance plethysmograph, was used in this study since it is the measure of segmental conductive volume that is independent of changes in cell membrane capacitance.

The rhesus volume changes were found to be consistent with those measured in humans during past experiments [13], however the squirrel monkeys were found to respond somewhat differently and in unexpected ways to HUT and HDT orthostatic stress. During HUT, one would expect the calf and thigh R-values to decrease and the upper and lower torso $R$ values to increase as body fluids drained from the torso segments into the calf and thigh. The opposite trends would be expected during HDT. These results were found in all Rhesus monkey segments and in the abdominal and thoracic segments of the squirrel monkeys.

The squirrel monkey's abdominal and thoracic volume changes seem to be similar to those found in the Rhesus monkeys while the responses of their lower body segments are quite different. The squirrel monkeys lost fluid from their calf, thigh, and pelvic segments during both the HUT and HDT test sequences. In addition, the volume changes found in the Rhesus monkeys are graded with increased tilt angle, while those of the squirrel monkeys are less dynamic 
at 5 and 10 degrees tilt and much more so at 20 degrees. Other, less dramatic, differences may exist between the two species at low angles of tilt, such as the transient response observed in the leg segments following 5-degree head up and head down tilt.

In an attempt to explain these unexpected results, we conducted several tests in which we measured calf and tail volume changes in the squirrel monkey during HUT or HDT.

The monkey's tails were placed parallel to their legs during the following test sequence:

$\begin{array}{ll}0-5 \mathrm{~min} . & \text { Horizontal } \\ 5-10 \mathrm{~min} . & \text { HUT or HDT at } 10 \mathrm{deg} . \\ 10-15 \mathrm{~min} . & \text { Horizontal } \\ 15-20 \mathrm{~min} . & \text { HUT or HDT at } 20 \mathrm{deg} . \\ 20-25 \mathrm{~min} . & \text { Horizontal. }\end{array}$

Figure 3 illustrates the results of those tests on three squirrel monkeys. During HDT, they initially lost fluid from their tails (as would be expected). In addition, the calf increased in resistance (decreased volume) also as expected. The tail exhibited an opposite effect; decreased $\mathrm{R}_{0}$, which means an increase in fluid volume. When placed at -20 deg. their calves continued to lose fluid and their tails gained volume. This would not be expected since the tail was placed parallel to the legs. During HUT, the squirrel monkey lost fluid from their tails during the total test sequence.

These results may have been due to a vasomotor response whereby the squirrel monkey uses their tail to help maintain a more constant upper body volume during postural changes. Such a response may also vary (as shown in Figure 3) between different monkeys tested. This would seem to be a viable mechanism considering that the squirrel monkey has two large veins running the length of his tail and uses his tail as a thermoregulatory device [12] and as an aid in keeping their balance during ambulation [9].

\section{Limitations}

Several factors should be considered when reading and interpreting the results of this study. First, a limited number of monkeys of each specie were tested during the head-up and head-down tilt tests. The results represent the segmental volume changes under a limited number of postural or position changes. They may be different during other environmental conditions, particularly in free-range animals in their natural environments.

In addition, two types of impedance electrodes were used on the Rhesus and squirrel monkeys. Regular ECG electrodes were used on the Rhesus monkeys after shaving and proper skin preparation. However, it proved difficult to use the same type of electrodes on the squirrel (particularly on the tail) due to their small segment size.
This difference may have yielded different results if we had been interested in actual quantitative values of segmental volume and blood flow in units of $\mathrm{ml}$ and $\mathrm{ml} / 100$ ml-min, respectively. However, as shown in Figures 1, 2, and 3 we were only interested in measuring and comparing the relative percent volume changes in each specie/ segment during HUT and HDT. The relative changes should not be affected by electrode type as long as the same type was used on a given specie during each test sequence.

\section{Acknowledgements}

The authors wish to acknowledge and thank Dr. Leon E. Kazarian and Dr. Beverley Girten for their assistance in conducting the study and helpful advice and guidance during the preparation of this manuscript.

\section{Conflict of Interest}

Neither of the authors have received or will receive any compensation or monetary benefit from the publication of this article. This article represents new research and has not been published elsewhere.

\section{Disclaimer}

The opinions or assertions contained herein are the private views of the author, and are not to be construed as official, or as reflecting true views any funding agency. Research was conducted under an approved animal use protocol in an AAALAC accredited facility in compliance with the Animal Welfare Act and other federal statutes and regulations relating to animals and experiments involving animals and adheres to principles stated in the Guide for the Care and Use of Laboratory Animals, NRC Publication, 2011 edition.

\section{Disclosure of Funding}

The experiments described in this article were, in part, funded by the USAF Harry G. Armstrong Aerospace Medical Research Laboratory, Biodynamics Effects Branch Project Job Order No. 2312V601 and, in part, by SBIR Grants 1 R43 HL074524-01 and 2 R44 HL074524-02A2.

\section{References}

1. Heiney SA, Blazquez PM. Behavioral responses of trained squirrel and rhesus monkeys during oculomotor tasks. Exp. Brain Res. 2011;212(3):409-416. https://doi.org/10.1007/s00221-011-2746-4

2. Friedman $\mathrm{H}$, Ator $\mathrm{N}$, Haigwood N, Newsome W, Allan JS, Golos TG, et. al. The critical role on nonhuman primates in medical research. Pathog. Immun. 2017;2(3):352-365.

3. Abbott $\mathrm{DH}$, Abee CR, Fairbanks LA, Kaplan JR, Marthas ML, Mathieson B, et. al. Demands for rhesus monkeys in biomedical research: A workshop report. ILAR Journal 2003;44(3):222-238. https://doi.org/10.1093/ilar.44.3.222 
4. Abee CR. Squirrel monkey (Saimiri spp.) research and resources. ILAR Journal 2000;41(1):2-9. https://doi.org/10.1093/ilar.41.1.2

5. Brady AG. Research techniques for the squirrel monkey (Saimiri sp.) ILAR Journal 2000;41(1):10-18. https://doi.org/10.1093/ilar.41.1.10

6. Spence JD, Pesout J, Melmon KL. Effects of antihypertensive drugs on blood velocity in rhesus monkeys. Stroke 1977;8(5):589-594. https://doi.org/10.1161/01.str.8.5.589

7. DeWeese J. Changes in blood pressure and heart rate during fixed-interval responding in squirrel monkeys. J. Exp. Ana. Behavior 2009;92(3):379-385.

https://doi.org/10.1901/jeab.2009.92-379

8. Schmidt M. Locomotion and postural behaviour. Adv. Sci. Res. 2010;5:23-39.

9. Young JW, Russo GA, Fellmann CD, Thatikunta MA, Chadwell BA. Tail function during arboreal quadrupedalism in squirrel monkeys (Saimiri boliviensis) and tamarins (Saguinus oedipus). J. Exp. Zool. 2015;999A:1-11. https://doi.org/10.1002/jez.1948
10. Matsunami K. Squirrel monkeys in space motion sickness. Japanese J. Physio. 2002;52:1-20.

11. Nyboer J. Electrical impedance plethysmography. Springfield, IL. Charles C. Thomas, 1970.

12. Fuller CA, Sulzman FM, Moore-ede MC. Circadian control of thermoregulation in the squirrel monkey, Saimiri sciureus. Am. J. Physiol. 1979;236(3):R153-R161. https://doi.org/10.1152/ajpregu.1979.236.3.r153

13. Montgomery LD, Parmet AJ, Booher CR. Body volumechanges during simulated microgravity:Auditory changes, segmental fluid redistribution, and regional hemodynamics. Ann. Biomed. Eng. 1993;21:417-433. https://doi.org/10.1007/bf02368634 